\title{
Teoría sociológica clásica y discapacidad: algunos apuntes para un diálogo en potencia
}

\author{
The classical sociological theory and disability: some notes for a \\ potential dialogue
}

\begin{abstract}
Resumen
El siguiente escrito plantea las posibilidades de establecer un diálogo emergente y necesario entre la temática de la discapacidad y marcos conceptuales clásicos de la teoría sociológica, bajo la sospecha de que en las obras de los teóricos reconocidos como 'clásicos', podemos encontrar explicaciones/comprensiones que tributen utilidad al entendimiento de la discapacidad, su reconceptualización y redefinición. Asumimos que la discapacidad es un fenómeno que se erige como complejo y multidimensional, y que la sociología nos permite verlo, por un lado, como un vaivén entre los constreñimientos estructurales que restringen las posibilidades de "existencia social" en los individuos con esta condición, y por otro lado desde la intencionalidad, sentido y motivaciones a partir de las cuales estas personas significan sus acciones, su condición asignada y su posición social. En este sentido, el siguiente artículo se propone desarrollar la siguiente pregunta: ¿cómo pueden las teorías sociológicas clásicas y sus conceptos contribuir a la comprensión de la discapacidad? Solo a modo de contextualización se ofrecen algunos datos empíricos provenientes de diversas fuentes.
\end{abstract}

\section{Palabras clave}

Sociología clásica, discapacidad, estructura, acción social.

\begin{abstract}
The following writing raises the possibilities of establishing an emergent and necessary dialogue between the subject of disability and the classical conceptual frameworks of sociological theory. We consider such possibilities under the suspicion that, in the works of theorists recognized as "classical", we can find explanations/ understandings that usefully contribute to the understanding of disability, its reconceptualization and redefinition. We assume that disability is a phenomenon that stands out as complex and multidimensional. We also assume that sociology allows us to see it, on one hand, as a sway between the structural constraints that restrict the possibilities of "social existence" in individuals with this condition, and on the other hand the intentionality, meaning and motivation from which these people signify their actions, their assigned status and their social position. In this sense, the following article sets out to develop the following question: How can classical sociological theories and its concepts contribute to the understanding of disability? By way of contextualization, some empirical data from various sources is offered.
\end{abstract}

\section{Keywords}

Classical sociology, disability, structures, social action.

\author{
Diego Solsona Cisternas \\ <diego.solsona@live.cl>
}

Universidad de Los Lagos. Chile

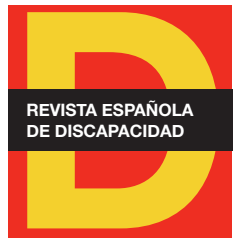

Para citar:

Solsona, D. (2020). "Teoría sociológica clásica y discapacidad: algunos apuntes para un diálogo en potencia". Revista Española de Discapacidad, 8(I), pp. 7-24.

Doi: <https://doi.org/10.5569/23405104.08.01.01>

Fecha de recepción: 03-06-2019 Fecha de aceptación:16-04-2020

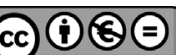




\section{Introducción ${ }^{1}$}

Este trabajo se articula a partir de la revisión de conceptos provenientes de diferentes autores clásicos que, desde diversas posiciones en la historia de las ideas de la teoría sociológica y sus concomitantes paradigmas, nos invitan a mirar (objetivar) la discapacidad como un fenómeno sociocultural moderno. Por lo mismo, iniciamos este artículo identificando algunos modelos que han definido e intervenido la discapacidad como objeto, proponiendo que la categoría discapacidad también puede ser vista como un fenómeno sociológico. En un primer momento se intenta establecer la relación entre discapacidad y lineamientos teóricos catalogados como "estructurales" (Marx, Durkheim y parcialmente Bourdieu), en un segundo momento se pretende plasmar un dialogo entre discapacidad y orientaciones teóricas que reivindican "la subjetividad en la acción social" (Weber, Schütz y Luckmann) para finalizar con un tercero en donde se plantean emergencias teóricas rotuladas como "intermedias" donde consideramos a Giddens y a Archer; a pesar de que ambas perspectivas se diferencian conceptualmente, las dos encajarían en la categorización de teorías intermedias.

Vale destacar que no es el objetivo de este escrito "tomar una posición" con algún autor o corriente específica sino, más bien, ofertar una amplia gama de conceptos y autores clásicos para entender la discapacidad, amplificando de esta forma los prismas y miradas a partir de los cuales los estudiosos del tema se pueden aproximar a él. También es importante explicitar que operacionalmente nos circunscribimos a la idea de que la discapacidad es esencialmente una "experiencia habitada", como dice Valenzuela (2015: 58) "los sujetos deconstruyen y reconfiguran las estructuras y representaciones sociales conforme a sus vivencias y desde distintos campos cognitivos, valorativos y afectivos para conducirse dentro de lo social", lo que incluye las estructuras que lo constriñen.

Finalmente, y solo a modo de ejemplificación empírica sobre la realidad de las personas con discapacidad, se exhiben algunos datos estadísticos provenientes del segundo estudio nacional de la discapacidad en Chile (Servicio Nacional de la Discapacidad, SENADIS, 2015) y otros a nivel mundial, extraídos de organismos internacionales como la Organización de las Naciones Unidas (ONU), la Organización Internacional del Trabajo (OIT) y la Organización de las Naciones Unidas para la Educación, la Ciencia y la Cultura (UNESCO).

\section{La discapacidad como fenómeno sociológico}

En este trabajo solo profundizamos en los modelos médico-rehabilitador y social, debido a que son los más usados en investigación e intervención de personas con discapacidad. Tal como dicen algunos autores (Imrie, 2004; Oliver, 1990) el modelo social de la discapacidad ha tendido a dominar la investigación y la escritura sobre el tema, y tiende a concebir a las personas como víctimas de una opresión causada por factores sociales y ambientales. Por su parte, Imrie (2001) asegura que los discursos de la discapacidad están inscritos en el contexto del dominio del modelo médico, el cual concibe esta condición como anormal, desviada y reducible exclusivamente a dificultades físicas y mentales.

1. Agradecimientos: al Profesor Doctor Oscar Mac-Clure por sus consejos y sugerencias para terminar este trabajo. El autor también agradece a la Agencia nacional de investigación y desarrollo (ANID) por el apoyo brindado para el financiamiento de sus estudios doctorales, a través de la beca de doctorado nacional $\mathrm{N}^{\circ} 21180221$. 
La discapacidad como objeto de estudio e intervención ${ }^{2}$ ha sido históricamente monopolizada y aprehendida por lo que se denomina el "modelo médico rehabilitador" (Romañach y Palacios, 2008), el cual caracteriza la discapacidad como una condición permanente de enfermedad. Lo anterior tiene como efecto que las personas con discapacidad son identificadas como un "conjunto de síntomas", que constituyen una tragedia personal y una situación indeseable, y cuya única forma de enfrentar y paliar sus efectos es el sometimiento a tratamientos médicos de rehabilitación (que, por supuesto, involucran hábitos de medicalización) con el fin de "normalizar sus cuerpos".

Como efecto del poder-saber médico, el Estado incorpora la visión de la medicina sobre la discapacidad como "legítima" (Ferrante y Ferreira, 2011). Tal como dice Ferrante (2008) este modelo convierte a las personas con discapacidad en sujetos susceptibles de ser intervenidos a través del asistencialismo y la caridad. No obstante, el modelo médico rehabilitador en los últimos años ha perdido parcialmente su piso epistemológico ante la irrupción del "modelo social de la discapacidad"3.

El modelo social de la discapacidad ha sido definido, abordado y tensionado por diversos autores (Romañach y Palacios, 2008; Míguez, 2014; Ferrante, 2008; Ferreira y Rodríguez Caamaño, 2008; Almeida et al., 2008). Estos autores coinciden en que la discapacidad es un efecto de la interacción entre una persona y su contexto; esto implica evitar buscar explicaciones dentro del individuo (como unidad atomizada) como si fuera responsable de su condición y de los efectos asociados a ella. Más bien se entiende que las causas de la discapacidad se encontrarían en el entorno, en las calles, en las disposiciones y diseños de las infraestructuras, en las políticas públicas del Estado y en las actitudes sociales discriminatorias de personas sin discapacidad (Solsona et al., 2018).

Con la irrupción del modelo social en la arena de la discapacidad, es que se produce una apertura para que la sociología se apropie del tema. Específicamente desde la sociología se propone que la discapacidad es una construcción social antes que una realidad estrictamente biológica (Abberley, 1987; Oliver, 1990). En este sentido, la discapacidad sería consecuencia de la interacción entre un individuo y su entorno, es decir, se destaca su carácter "relacional"; en términos coloquiales podemos decir que la discapacidad se produce cuando los individuos interactúan socialmente y son conscientes de los obstáculos y barreras que son efectos de las propias creaciones y disposiciones sociales.

Según Pérez y Chhabra (2019: 13) "el modelo social británico de la discapacidad reconoce que un impedimento no es lo mismo que una discapacidad. El primero es individual y privado, la segunda es estructural y pública (...) El modelo social no atribuye la discapacidad al individuo, sino al entorno, y reivindica un cambio social”. Este cambio podría darse en el contexto de un proceso de morfogénesis (Archer, 2009), el cual desarrollaremos más adelante, pero, a grandes rasgos, implica cambios en los sistemas sociales a partir de la apropiación que hacen los individuos de las propiedades que pertenecen a ese sistema ${ }^{4}$.

\footnotetext{
2. En este artículo solo se discuten el modelo médico-rehabilitador y el modelo social. Se consideraron por ser los modelos más utilizados en estudios e intervenciones sobre el tema. Por ejemplo, la definición de la Clasificación Internacional del Funcionamiento define la discapacidad de forma interaccionista como una "deficiencia" (modelo médico) que interactúa con un entorno hostil, mal diseñado y no adaptado (modelo social) para las personas con esta deficiencia (OMS, 2001).

3. Existen otras tipologías y modelos sobre la discapacidad que han tenido mucho auge en las últimas décadas, tales como: modelo tradicional, modelo de la diversidad funcional, modelo post-humanista, modelo minoritario, modelo biopsicosocial, modelo relacional, modelo de los derechos humanos, modelo cultural. Véase Pérez y Chhabra, 2019.

4. En este caso no nos referimos a la discapacidad como un sistema, sino que más bien entendemos por sistema las estructuras del Estado a partir de las cuales se interviene la discapacidad como problema social, político y cultural.
} 
Lo anteriormente planteado se pone en evidente contraste con el modelo biologicista de la lógica individual, el cual tiene su base en el modelo médico hegemónico ya explicado anteriormente. De acuerdo con Brogna (2006: 2), la discapacidad "no es una condición a curar, a completar o reparar, es una construcción relacional entre la sociedad y un sujeto (individual o colectivo) (...) La discapacidad toma cuerpo en un espacio situacional, dinámico e interactivo entre alguien con cierta particularidad y la comunidad que lo rodea". En esta misma línea Ferreira y Rodríguez Caamaño (2008:144) definen la discapacidad en un contexto más acotado, vinculado con las temáticas de exclusión y marginación presentes en nuestras sociedades; ellos prefieren hablar de un "entorno incapacitante, es decir, como un colectivo segregado por el conjunto de la comunidad, homogeneizado por su insuficiencia, su incapacidad y su valía reducida respecto a las suficiencias, capacidades y valías de la generalidad de la población no discapacitada".

Gómez Tagle y Castillo (2016) plantean que la sociología de la discapacidad es una especialidad reciente, y llama la atención el hecho de que la disciplina no haya considerado la discapacidad como un objeto de estudio plausible y frecuente para la investigación sociológica, a pesar de su relevancia teórica y demográfica. Los autores plantean que la intromisión o apropiación del tema por parte de la sociología puede conducir eventualmente a la construcción de un nuevo paradigma e incluso a propiciar elementos para la creación de políticas públicas más eficaces y que dignifiquen las condiciones de existencia de las personas con discapacidad. Si bien este ensayo no tiene esas pretensiones normativas, sí nos parece que la sociología "tiene algo que decir" con respecto a la discapacidad, asumida como una producción o fabricación históricasocial de sujetos y grupos. Y que, por lo tanto, no considerar como primera referencia a los clásicos de la sociología sería, por decirlo de alguna manera, desechar arbitrariamente unos 150 años de producción y vigencia de mediadores conceptuales impregnados de potencial analítico (explicativo y comprensivo) para abordar el fenómeno.

Asumiendo que la discapacidad es un fenómeno sociológico, es importante reconectar el tema con nociones teoréticas y mediadores conceptuales considerados como "clásicos"; en un tiempo donde las certezas teóricas parecen perder su potencial explicativo, y considerando que hay abundante literatura sobre el tema, se hace necesario vincular la discapacidad como objeto de estudio, con nociones teóricas de fondo. La teoría sociológica eventualmente podría aportar a reconceptualizar y problematizar los enfoques existentes sobre la discapacidad.

\section{Primer momento "Enfoques estructuralistas y discapacidad"}

\subsection{La discapacidad como hecho social}

Solo a modo de contextualización, es importante plantear que la discapacidad se ha entendido de forma hegemónica como una "enfermedad" del cuerpo, lo cual constituye una tragedia individual que hace de los individuos que la portan personas anormales, patológicas y con menor valor en comparación a las personas sin discapacidad. Al mismo tiempo son percibidas como susceptibles de ser intervenidas e institucionalizadas en los ámbitos médicos y depositarias de políticas públicas compensatorias por parte del Estado (Solsona et al., 2018; Míguez, 2014; Ferrante, 2008; Ferreira y Rodríguez y Caamaño, 2008; Almeida et al., 2008). 
Considerando lo anterior, podemos introducir la clásica noción de Durkheim sobre los "hechos sociales". Para Durkheim (2000:38-56) estos son "externos al individuo, son anteriores a él, son coercitivos y obligatorios, se fundamentan en el derecho y las costumbres y son aprendidos y aprehendidos en las distintas etapas de la socialización. También son modos de actuar, de pensar y de sentir que existen fuera de las conciencias individuales, se imponen a nosotros lo queramos o no".

En este sentido la discapacidad es un "hecho social", porque naturaliza esa condición en el sentido de que es biológica. Posteriormente es aprehendida e incorporada por las estructuras socioculturales que reproducen esta naturalización simbólicamente, además de que modelan y construyen a las personas con discapacidad como "sujetos diferenciados". Por ejemplo, en las escuelas en Chile existen programas de integración escolar (PIE) para estudiantes con discapacidad, no obstante, el discurso de la integración, paradójicamente, promueve ámbitos diferenciados para los estudiantes con discapacidad; son evaluados de manera diferente (adaptaciones curriculares), tienen otros espacios, como salas especiales en donde los separan del resto de sus compañeros, otros horarios, y son intervenidos por otros profesionales que los "corrigen" tales como: fonoaudiólogos para los trastornos del lenguaje, psicopedagogos para los retrasos de aprendizaje, etc. Es decir, considerando que los hechos sociales son aprehendidos en las etapas de la socialización, es en la escuela como institución donde los niños y niñas con discapacidad "aprenden a ser diferentes".

El ejemplo de la escuela se reproduce en otros ámbitos más macro, como por ejemplo el Estado como súper-estructura (Marx, 1857/1997) el cual construye a las personas con discapacidad como sujetos problemáticos y los interviene "diferenciadamente" confundiéndose muchas veces estas intervenciones, con caridad, altruismo y filantropía. Evidencia de esto es que la mayoría de los Estados modernos cuentan con agencias, servicios o fondos nacionales de discapacidad, las cuales son las encargadas de planificar y ejecutar políticas para las personas con discapacidad, políticas que generalmente se basan en la lógica de la compensación (ayudar a aquellos que son algo que no eligieron ser), los intervienen como "diferentes" (Rosato et al., 2009). O que la mayoría de los Estados certifiquen la discapacidad a través de diagnósticos biomédicos y psicosociales, entregando un carnet o credencial a las personas, lo que los convierte identitariamente en "otros", "diferentes", dependientes del Estado y sus bonificaciones.

En definitiva, la discapacidad como categoría social construida, y a partir de la cual se ciernen unas prácticas, símbolos y discursos asociados a los individuos que la portan, termina imponiéndose como hecho social, lo que provoca como resultado que las personas la "naturalicen" y tiendan a ser socializados como diferentes.

\subsection{Marx: discapacidad y capitalismo}

Por otro lado, no podemos desentendernos de la fuerza de las estructuras económicas. Como bien explica Marx (1857/1997) en su Introducción general a la crítica de la económica política, los individuos producen en sociedad, al mismo tiempo que estos individuos están producidos socialmente. En este sentido, la irrupción de un sistema capitalista de producción a partir del hecho histórico material de la revolución industrial crea un nuevo tipo de sociedad con sus respectivas dinámicas. Marx afirma "ninguna producción es posible sin un instrumento de producción, aunque este instrumento sea sólo la mano; ninguna, sin trabajo pasado 
acumulado, aunque este trabajo sea sólo la destreza que el ejercicio repetido ha desarrollado y concentrado en la mano del salvaje" (1857: 36). Asumiendo que la producción es el motor del sistema capitalista, la estructura económica como base de las relaciones sociales necesariamente debe jerarquizar unas funciones sobre otras, y es aquí donde nuevamente la estructura les propone un constreñimiento a las personas con discapacidad. Estas al ser percibidas como "anormales" o "enfermas", son también rotuladas como incapaces de ocupar siquiera una posición devaluada en los engranajes productivos capitalistas, es decir, no son funcionales al sistema productivo capitalista que pondera la eficiencia, destreza y rapidez como valores deseables y necesarios.

En concordancia con lo expuesto anteriormente, el capitalismo privilegia en su fase más ortodoxa, cuerpos fuertes, sanos, hábiles y capaces de producir, de trabajar una gran cantidad de horas diarias. De esta forma, la estructura productiva le impide a la persona con discapacidad ocupar una posición en la cadena productiva, lo que también deviene en una disminución de sus posibilidades de consumo y de inclusión social, en definitiva, de otorgarle sentido a una actividad convenidamente esencial del ser humano como lo es el trabajo. Esto se relaciona con la preminencia del modelo médico de la discapacidad que, como ya hemos dicho, cataloga a los cuerpos como enfermos e incapaces y, por lo mismo, los esfuerzos de rehabilitación tienen como objetivo, no que las personas con discapacidad sean incluidas en diversas actividades sociales, sino que sean funcionalmente productivas en el engranaje del sistema económico.

Pensamos que la irrupción y consolidación del capitalismo como sistema económico es un proceso histórico fundamental para situar la discapacidad. Según Ferrante y Ferreira (2011) las marcas de exclusión social de las personas con discapacidad no eran tan marcadas en las sociedades tradicionales pre-capitalistas, esto tiene que ver, según los autores, con que resulta "funcional" para la estructura socio-histórica, la lógica del beneficio demanda, fuerza de trabajo "productiva" y consumo (individual) "egoísta"; se trata de la permanente maximización económica de individuos en competencia. La exclusión de las personas con discapacidad, articulada en torno a ese habitus de grupo inducido, elimina "excedentes improductivos": mano de obra poco rentable, individuos poco competitivos (Oliver, 1990; Ferrante y Ferreira, 2011). Igualmente, Córdoba (2008) afirma que las personas con discapacidad al no ser consideradas como "productivas" son susceptibles de discriminación y exclusión.

Volviendo a Durkheim, en su texto sobre la división social del trabajo (1987) el autor plantea que una de las características fundamentales de la sociedad capitalista industrial, es la "especialización de las tareas". Cuando las tareas se jerarquizan, esto inmediatamente tiene como efecto situar a algunos individuos en una posición de privilegio, y a otros en una situación de exclusión. A pesar de las legislaciones en favor de la inclusión laboral de las personas con discapacidad, estas siempre son ubicadas en ocupaciones con bajos salarios, e integradas a una empresa o servicio, solo por la obligatoriedad de incluirlos como "sujetos diferentes", que no pueden elegir la mayor parte de las actividades que realizan.

Según los datos de la "Segunda encuesta nacional de discapacidad en Chile" (Servicio Nacional de la Discapacidad, SENADIS, 2015) solo un 39,3\% de la población adulta con discapacidad ocupa un puesto en el mercado laboral, un 57,2\% está inactivo y otro 3,6\% desempleado. Otro dato interesante revela que el promedio de un salario para las personas "sin discapacidad" en Chile es de 434.586 pesos, en cambio, la población con discapacidad tiene un promedio de salario evidentemente menor, 282.000 pesos aproximadamente (420 dólares promedio). Esto se condice con los datos a nivel mundial, según se estima, unos 386 millones de las 
personas en edad de trabajar tienen discapacidad, Organización Internacional del Trabajo (OIT, 2001). El desempleo de personas con discapacidad alcanza hasta un $80 \%$ en algunos países, y a menudo los empleadores suponen que estas personas no pueden trabajar (Organización de las Naciones Unidas, 2017).

El capitalismo como sistema económico de producción, a la luz de algunas observaciones e interpretaciones de sociólogos clásicos, demuestra ser un sistema que prescinde de las personas con discapacidad al no cumplir con los parámetros de eficiencia productiva deseados para su buen funcionamiento. Esto tiene como consecuencias diversas formas de exclusión y segregación que ponen en desventaja a las personas con esta condición.

\subsection{Bourdieu: discapacidad y habitus}

Pierre Bourdieu, uno de los sociólogos más leídos en los últimos 30 años, o para ser más justos, un verdadero clásico moderno, ha propuesto conceptos muy útiles que podrían aproximarnos a entender la discapacidad desde su dimensión estructural construida. En primer lugar el habitus definido como; "esquemas mentales y prácticos resultado de la incorporación de visiones y divisiones sociales objetivas que configuran principios de diferencia y pertenencia a ciertos campos, principios que por cierto son el resultado de una serie de disposiciones incorporadas en el curso de una trayectoria" (Bourdieu, 1979/1998: 18), también asegura que el habitus es producto de cómo los sujetos internalizan las estructuras, algo similar a los "hechos sociales" de Durkheim.

En este sentido las personas con discapacidad van construyendo su habitus en función de cómo son modelados por las estructuras y percibidos por aquellos que no están en condición de discapacidad, es decir, el Estado los ve como sujetos en "falta de algo", que deben ser compensados desde el asistencialismo, el sistema médico los ve como enfermos, las personas sin discapacidad los ven como personas dignas de lástima, etc. Esto deviene, por ejemplo, en situaciones de "autoexclusión": una persona con discapacidad sabe que no puede tomar un autobús que no tenga rampas para subirse, que no puede ir a un teatro que no esté adaptado espacialmente, o que no puede practicar ciertos deportes. Esto restringe particularmente las posibilidades de constituirse como sujetos participantes y significantes de la vida social, lo que igualmente produce como efecto una disminución de sus tipos de capitales.

Para Ferreira (2009) el habitus de la discapacidad se refiere al sentido de adquirir una discapacidad y a las prácticas esperables a esa condición, como una especie de interpretación de un "rol”. El sentido legítimo de la discapacidad es determinado por la ciencia médica para la cual un cuerpo en estas condiciones es un cuerpo "anormal". La designación de desviación implica que un cuerpo no es apto para las funciones que se otorgan al cuerpo "normal". Así, la conciencia de poseer un cuerpo "imperfecto" se presenta como una condición natural y suficiente para limitar a las personas en sus posibilidades de desenvolvimiento (Ferreira, 2009; Venturiello, 2010: 178). De hecho, la misma teórica argentina citando a Corker (2008) plantea que el concepto de habitus puede encontrar un camino fructífero para la superación de la dicotomía de la experiencia de la discapacidad como realidad objetiva y la vivencia subjetiva.

También Ferrante y Ferreira se refieren al habitus de la discapacidad como "estructura estructurada, producto histórico a través del cual el Estado inculca la concepción del cuerpo no legítimo definida por el campo 
médico; y como estructura estructurante determina el límite de lo pensable y lo no pensable y genera unas prácticas y unos juicios sistemáticos. (...) Sobre esa imposición, el colectivo queda marcado con las señas de la exclusión social” (2011: 90). Lo planteado por estos autores también coincide con lo propuesto por Solsona et al. (2018) quienes, inspirados en la teoría fenomenológica de los imaginarios sociales, reconocen que la discapacidad es un imaginario social proveniente de las definiciones biomédicas y que este es apropiado por el Estado, el cual lo convierte en ideología y termina aterrizando como políticas públicas de intervención de la discapacidad, caracterizadas principalmente por un exacerbado asistencialismo.

\subsection{Bourdieu: discapacidad y tipos de capital}

También el sociólogo francés define el capital social como "el agregado de los recursos reales o potenciales que se vinculan con la posesión de una red duradera de relaciones más o menos institucionalizadas de conocimiento o reconocimiento mutuo" (Bourdieu, 2000: 148). Asimismo, afirma que existe el capital económico (recursos financieros, bienes, propiedades, etc.) y el capital cultural (nivel educacional, estudios, viajes de turismo, visitas a museos, etc.). Todos estos son importantes para las posibilidades de participación e inclusión social de los sujetos y también para determinar su posición en la estructura social.

Siguiendo una secuencia lógica, una persona con discapacidad que no trabaja no puede percibir ingresos y, por lo tanto, no cuenta con un capital económico estable (al menos que perciba una pensión del Estado siempre menor a un salario de mercado). Una persona con discapacidad que no participa de instancias de socialización como reuniones, fiestas u otras, no puede portar un capital social, y es sabido que una red duradera de relaciones y contactos (Bourdieu, 2000) puede erigirse como un soporte de apoyo para estas personas, las cuales necesitan adaptaciones y otro tipo de recursos intangibles. Sin embargo, ellas mismas y especialmente sus cuidadores, prefieren adoptar la táctica de invisibilización y ocultamiento, es decir, para evitar los estigmas y miradas devaluadas de las que son objeto, prefieren replegarse en los ámbitos privados, en una especie de ostracismo doméstico; es un habitus que cuenta con la aquiescencia de las personas con discapacidad (Imrie, 2004).

Igualmente, prefieren no recorrer la ciudad y sus espacios porque este es el lugar por antonomasia en el que son "reconocidos" por su discapacidad, o mejor dicho "desconocidos" o juzgados como la excepción a la norma. Estos espacios están configurados arbitrariamente para aquellos que no tienen discapacidad. Como dice Simmel (1903/2005) en la metrópoli o ciudad moderna, los tiempos son rápidos, acelerados y rutinarios, en función de la vida productiva y comercial; esta también sería una razón para no participar en estos espacios, no solo porque son poco amigables en su diseño estructural, sino también por el rechazo simbólico que provoca tolerar a un grupo que requiere de otros tiempos, procesos y adaptaciones.

Situaciones como las mencionadas corroen la promoción de la confianza como condición necesaria para forjar relaciones duraderas capaces de traducirse en capital social. Según los datos del ya citado Servicio Nacional de la Discapacidad, SENADIS (2015) en Chile, un 50,9\% de personas con discapacidad no participa en organizaciones sociales porque su salud se lo impide; por otro lado, un $74,8 \%$ de personas con discapacidad declara haber asistido a algún evento cultural durante el último mes versus un $91,6 \%$ de personas sin discapacidad. 
Conectado con lo anterior, las posibilidades de tener un capital cultural institucionalizado, es decir, un título o diploma que certifique estudios superiores o profesionales, a través del acceso y egreso de la educación superior técnico-profesional (Bourdieu, 2000) son muy restringidas. Según la última encuesta nacional de la discapacidad (Servicio Nacional de la Discapacidad, SENADIS, 2015) solo un $15 \%$ de las personas con discapacidad llegan a estudiar a la educación superior y 2/3 de ellas desertan por falta de apoyo. Que una persona con discapacidad obtenga un título universitario o técnico superior para ponerlo en valor en el mercado laboral es una singular excepción. Es sabido que el capital cultural institucionalizado es uno de los principales factores que determinan la posición de los individuos en el espacio social (Ferrante y Ferreira, 2011; Bourdieu, 1991). Esto tiene un correlato a nivel mundial, según la UNESCO (Organización de las Naciones Unidas para la Educación, la Ciencia y la Cultura, 2013) un $90 \%$ de niños y niñas con discapacidad no asiste a la escuela y la tasa de alfabetización de personas con discapacidad a nivel mundial no alcanza el $3 \%$.

En definitiva, estructuras como las relaciones de producción, la escuela, el Estado, la clase, la ciudad y una abstracción como lo son los hechos sociales, restringen las posibilidades de las personas con discapacidad de ser sujetos. A pesar de que este escrito no es empírico, es importante mencionar que existen estadísticas que nos sugieren que la discapacidad en Chile se caracteriza por ser: empobrecida (situada en los quintiles más bajos de estratificación social por ingresos), con baja escolaridad (menor promedio de años en educación formal que la población sin discapacidad), con baja participación social, feminizada $(72,1 \%$ de personas con discapacidad son mujeres), que son usuarios de los servicios públicos, dependientes de las instituciones de salud, etc. La discapacidad se construye y, en el lenguaje de Bourdieu, se reproduce, en ámbitos institucionalizados que propician, en algunos casos explícitamente, la identificación de la discapacidad como algo devaluado, indeseado y problemático.

\section{Segundo momento "Acción social, subjetividad y discapacidad"}

Las personas con discapacidad son capaces de darle sentido a sus acciones y vivencias de forma individual, más allá de los rótulos u opresiones estructurales que se les pueden presentar. La inscripción de sus experiencias puede variar dependiendo de las trayectorias biográficas e individuales de los sujetos. Según Ferrante y Ferreira (2011: 90) "los modos de vivir la discapacidad variarán de acuerdo a las singularidades de la biografía de cada agente en particular". "Al mismo tiempo es importante porque, a pesar de las exclusiones multiformes a las que son vulnerables las personas con discapacidad, estas, en su condición de agentes, consiguen oponerse a la subordinación impuesta por unas condiciones degradantes. Desarrollan defensas individuales y colectivas, puntuales o duraderas, inscritas de modo perdurable en los habitus: el humor, la ironía, el "empecinamiento obstinado"; formas menospreciadas de resistencia (Bourdieu, 1999; Ferrante y Ferreira 2011: 98). Es decir, a pesar de la preminencia de una mirada que los señala como "objeto" tanto por parte del sistema medico como del Estado, las personas despliegan tácticas y estrategias en su vida cotidiana, muchas de ellas discursivas, con el objetivo de paliar parcialmente los efectos prácticos de la ilegitimidad que se le adjudica a sus cuerpos, y la exclusión multiforme a la que están expuestos. 


\subsection{Weber: discapacidad y sentido de la acción social}

Según Weber el mundo moderno capitalista ha entrado en una fase de profunda "racionalización", lo cual no solo ha instrumentalizado las relaciones económicas en el sistema productivo, sino que también ha aterrizado en la vida cotidiana de las personas. De acuerdo con el teórico alemán, la comprensión de la acción social consiste en la captación interpretativa del sentido o conexión de sentido de las acciones humanas. A partir de esto, elabora su modelo con 4 tipos ideales de acción; (1) con arreglo a fines (instrumental), (2) con arreglo a valores (ponderada en el puro mérito del valor), (3) afectiva (emotiva) y (4) tradicional (legitimación de una práctica, casi reactiva) (1922/ 2002: 28-34).

Es pertinente interrogarse hasta qué punto las personas con discapacidad pueden significar sus acciones en aras de darle sentido. Por ejemplo, cuando una persona con discapacidad es sometida a un tratamiento de rehabilitación y medicalización, se produce una pugna de sentidos. Como bien señala Ferrante (2009), por un lado, los profesionales de la salud pretender corregir, normalizar y rehabilitar los cuerpos con el objetivo de que "funcionen adecuadamente", sobre todo dentro del contexto de producción capitalista. No obstante, las personas con discapacidad pueden orientar su acción no solamente desde la perspectiva de lo instrumental, sino que eventualmente involucran expectativas impregnadas de lo emotivo y afectivo, en donde estos procesos clínicos son para ellos experiencias cargadas de riesgos e incertidumbres, al mismo tiempo que asumen que los resultados esperados al realizar estos tratamientos, podrían contribuir a aumentar su participación social, familiar, a dejar de ser dependientes, a ser autónomos, libremente móviles, etc.

Podemos decir que, en las personas con discapacidad, un tratamiento de rehabilitación puede ser una "acción con arreglo a fines" (rehabilitarse) y una "acción afectiva" (participar con otros, establecer vínculos emocionales, ser reconocidos en sus derechos, etc.). Los profesionales de la salud no siempre alcanzan a ver los plexos de sentido involucrados en las intervenciones, sentidos que trascienden la acción con arreglo a fines, para situarse en una acción afectiva-emotiva, es decir, en términos más coloquiales, las personas con discapacidad no van a un hospital, centro de atención primaria o centro de rehabilitación solamente para ser "curadas" o "tratadas", asisten porque quieren participar, quieren ser reconocidas e incluidas, y quieren que se les trate de acuerdo a la normativa vigente y al sentido común de la empatía.

\subsection{Weber: discapacidad, dominación y legitimidad}

Igualmente es importante rescatar en Weber los conceptos de dominación y legitimidad, donde también hay constreñimientos estructurales que dialogan recursivamente con las posibilidades de existencia social de las personas con discapacidad. Weber entiende la dominación como "la probabilidad de encontrar obediencia a un mandato de determinado contenido entre personas dadas" (Weber, 1922/2002:171). El modelo médico rehabilitador, justamente, ejerce dominio sobre los cuerpos de las personas con discapacidad, debido al saber-poder experto que ostentan (Rosato et al., 2009; Foucault, 2007); esto implica que las personas con discapacidad terminan "legitimando" (por los argumentos científicos racionales del entrenamiento y quehacer del médico) su rol como "permanentes enfermos", produciéndose una amalgama entre un tipo de dominación racional (el medico sabe y trabaja dentro de una institución reconocida) y otro de dominación tradicional (la creencia en la tradición medica) es decir, "si el medico dice que necesito tratamientos de rehabilitación y medicalización...debe ser así”. 
Pero, en definitiva, estamos principalmente frente a un tipo de dominación racional-legal, o sea, una especie de sometimiento (no necesariamente involuntario e inconsciente) a las ordenanzas estatuidas en el saber del médico, quien es validado por ser miembro de una institución médica (hospital, clínica, centro de rehabilitación, etc.) y por su capital cultural institucionalizado (un título universitario), por lo tanto, la dominación descansaría en la validez racional científica, casi sacralizada e incuestionable de los procedimientos, tratamientos y acciones de los médicos, lo cual hace prever para sus pacientes, los mejores y más efectivos resultados esperados.

A partir de la teoría sociológica del sentido de la acción social de Weber, no solamente podemos ver que es posible interpretar los motivos y significados que le dan las personas con discapacidad a sus acciones, sino que también se puede identificar cómo la legitimación de ciertas prácticas médicas propias de un modelo hegemónico de intervención de la discapacidad producen una dominación casi natural hacia las personas con discapacidad, quienes convencidos de la validez racional científica del poder saber-medico, se "entregan" a los tratamientos clínicos, confiando en que sus condiciones de salud y existencia en general, mejorarán a partir de estos.

\subsection{Schütz y Luckmann: discapacidad y mundos de la vida}

Otros teóricos vinculados a una sociología que reivindica la subjetividad de la acción social son Schütz y Luckmann (2003), quienes en su obra sobre "las estructuras del mundo de la vida" definen a este mundo como "pre-científico, acotado y pragmático, al mismo tiempo que lo experimentamos como incuestionable, cotidiano y natural, y en este ámbito podemos ser comprendidos por nuestros semejantes y solo en él podemos actuar con ellos" (2003: 25-28). Sin embargo, dentro de lo presupuesto en la actitud natural de este mundo existen algunas premisas de las cuales se puede sospechar que las personas con discapacidad quedarían excluidas del cumplimiento de las mismas. De partida, compartir sentidos es una experiencia innegablemente "sensorial" y los autores citados hablan de que la construcción de intersubjetividad (crear sentidos colectivamente) es posible gracias a que los individuos asumen que están rodeados de otros que tienen un cuerpo y conciencia esencialmente igual a las suyas.

Lo anterior es al menos suspicaz; por ejemplo, un estudio en la Patagonia chilena evidenció que los cuerpos con discapacidad son construidos desde el imaginario de la incompletud (Solsona, 2017), es decir, se les percibe como "en falta de algo", por lo tanto, serían incapaces de encontrar sentidos con otros, ya que no tienen un cuerpo esencialmente igual a sus semejantes (personas ciegas, sordas, con movilidad reducida). Además, también es cuestionado el sentido de realidad y conciencia de las personas con discapacidad intelectual o mental (peyorativamente captados como locos, deficientes, desviados, anormales, infantilizados, hipersexuados, etc.), o sea, tampoco sus conciencias serían similares a las de sus semejantes, lo que tiene como efecto dificultades para la construcción de significados y sentidos compartidos.

Podemos plantear que el mundo de la vida como escenario de la vida cotidiana y sus estructuras de sentido son más complejas de captar y experimentar para las personas con discapacidad. Las personas con discapacidad tienen códigos diferentes, pensemos en las personas sordas que utilizan la lengua de signos, o las personas ciegas que utilizan el sistema braille, su única posibilidad de construir intersubjetividad con otros depende necesariamente de que las demás personas manejen sus códigos comunicacionales. Igualmente, 
las personas con movilidad reducida, al estar sumergidas en espacios de confinamiento (Imrie, 2004) y por la misma fragmentación de sus cuerpos, tienden a ser vistas como estáticas o inmóviles, lo que deviene en una restricción de sus movilidades y, por ende, en una disminución en sus posibilidades de socializar e interactuar. Podemos decir que quien no tiene experiencias de interacción frecuentes está menos expuesto al mundo sensible y, por lo tanto, su propio mundo es menos legible, comprensible e intersubjetivo.

Con Weber, Schütz y Luckmann hemos podido orientarnos hacia un entendimiento de las personas con discapacidad desde un prisma más subjetivo, no obstante, una vez hechos los paralelos entre algunos conceptos planteados y la realidad vivida de las personas con discapacidad, sugiero que los sentidos y significados de la acción son principalmente permeados por la construcción social que se ha hecho de ellos y por las formas de dominación naturalizadas por el modelo médico bajo la etiqueta de la "enfermedad", ante lo cual, y por el momento, tendríamos que decir que en el caso de las personas con discapacidad, los sentidos y significados otorgados a sus acciones, son al menos diferenciados.

\section{Tercer momento "Emergencias teóricas intermedias y discapacidad". Intentando conciliar la estructura y la acción social con sentido}

\subsection{Giddens: discapacidad, propiedades y dualismo estructural}

Giddens (1984/2011) pone especial énfasis en la reflexividad de los actores sociales sobre sus roles y posiciones en las estructuras sociales, aunque el afirma al igual que Archer (2009) que es mejor hablar de "propiedades estructurales", las cuales son aspectos institucionalizados que ofrecen solidez en un tiempo y espacio. En este contexto, los agentes utilizan los recursos del poder para reproducirlos en el curso de una interacción, lo que Giddens denomina "dualismo estructural" (Giddens, 1984/2011: 3-75).

Un buen ejemplo de reflexividad y apropiación de las propiedades estructurales por parte de las personas con discapacidad es el caso de las políticas públicas sobre discapacidad.

Por ejemplo, en Chile, el Estado como estructura se convirtió en medio y resultado de cambios y modificaciones tendientes a la inclusión de personas con discapacidad a partir de la creación del Servicio Nacional de la Discapacidad (SENADIS), órgano que se crea después de que el Estado chileno se adhiriera a la Convención de derechos para las personas con discapacidad de la ONU en el año 2008. El Estado adopta una política para aumentar la participación a través de instancias de consultas ciudadanas para que las personas con discapacidad sean incluidas en la formulación y ejecución de políticas públicas que los involucran. Las personas empiezan a ser convocadas para "reflexionar, diagnosticar y proponer programas y políticas públicas". Su reconocimiento como actores clave en la construcción de políticas públicas deviene en una apropiación de la nueva institución y, efectivamente, se crean políticas que apuntan a capacitar a la sociedad civil, a los cuidadores, a los profesionales de la salud y a los profesionales del área social y jurídica, en temas relacionados con lenguaje inclusivo, formas de tratar a las personas, nuevos beneficios sociales, mayor participación social y otros orientados a la consecución de la autonomía de las personas con discapacidad. 
Lo anterior revela cómo las personas con discapacidad podrían pasar de ser concebidas e intervenidas como enfermas a ser tratadas como ciudadanas y ciudadanos con derechos, esto en el entendido de que efectivamente el Estado oriente sus acciones a la consecución de los objetivos de inclusión. Además, capacitar a la sociedad civil sin discapacidad, a funcionarios públicos, de la salud y el poder judicial, promulgar leyes tendentes a modificar las infraestructuras físicas y arquitectónicas que obstaculizan la participación socio-espacial de las personas con discapacidad, son sin duda acciones enmarcadas en el entendimiento de que la discapacidad es una situación "preminentemente social" y no médica, por lo cual, realizar transformaciones y adecuaciones sociales parece ser el camino para avanzar en la inclusión de las personas. En definitiva, son acciones que demuestran parcialmente una adherencia al paradigma del modelo social de intervención de la discapacidad.

A partir de estas nuevas políticas, es interesante observar cómo las personas se convierten en sujetosagentes, es decir, se enfatiza la capacidad creativa del agente a partir de la condición reflexiva de las prácticas cotidianas (Venturiello, 2010; Ferreira, 2009), apropiándose de la estructura que los define e interviene (en este caso el Servicio Nacional de la Discapacidad del Estado de Chile).

Las personas con discapacidad utilizan los recursos ofertados no solamente para mejorar ciertas condiciones en su calidad de vida sino también para participar socialmente y ser reconocidos como sujetos de derechos. Incluso, eventualmente, pueden llegar a "empoderarse" a partir de la creación de organizaciones comunitarias y territoriales, sociales o privadas, postulando a fondos concursables (proyectos de emprendimiento productivo, conseguir ayudas técnicas o tecnologías asistivas, recibiendo fondos para mejorar barrios y viviendas, etc.). También pueden fortalecer la articulación estratégica de sus organizaciones, asistiendo a las capacitaciones que ofrece el Estado en liderazgo, técnicas de comunicación, formulación y evaluación de proyectos, etc. Desde esta perspectiva la discapacidad se erige, según señala García-Alonso (2003) como un modo en que los individuos se perciben como sujetos y son llevados a reconocerse como colectivo.

\subsection{Archer: discapacidad y dualismo analítico}

Finalmente, Margaret Archer (2009) y su teoría del dualismo analítico probablemente constituye la aproximación teórica más contundente con respecto a una asociación entre la estructura y la acción, y esto también aplica a la discapacidad. Dos de los conceptos más importantes en Archer son el de "morfoestasis", entendido como los procesos que tienden a mantener la forma del sistema social, y la "morfogénesis" que son los procesos que tienden a cambiar una forma en el sistema social.

En el caso de la discapacidad, la morfoestasis, es decir, el statu quo a partir del cual se entiende y se interviene la discapacidad, se traduciría en las estructuras opresivas existentes en la sociedad tales como: diseños urbanos poco accesibles, institucionalización médica, políticas públicas del Estado asistencialistas, etc. Todas estas acciones tienden a reproducir el imaginario dominante y los estereotipos que construyen a las personas con discapacidad como incapaces, enfermas, susceptibles de beneficencia y dignas de conmiseración. No obstante, Archer afirma que no hay estructura sin sujeto, o mejor dicho, la estructura solo se valida en función del significado y sentido que los sujetos le otorgan. Sin embargo, lo peculiar del caso de la discapacidad es que emerge lo que Archer llama una "complementariedad necesaria", "esto significa 
que los sujetos al apropiarse de la estructura la legitiman en la medida en que son capaces de utilizarla y de reconocer su rol dentro de la misma, sin la necesidad de renunciar a su individualidad” (2009: 273).

Por ejemplo, el modelo médico rehabilitador como "sistema de intervención de la discapacidad funciona como condicionamiento estructural que tiene como lógica situacional una protección de este modelo". En términos más coloquiales decimos condicionamiento estructural, porque las personas con discapacidad están obligadas a asistir a los centros médicos para ser tratadas, no solamente a intervenciones clínicas, ya que el dominio de este modelo también implica, por ejemplo, que si una persona quiere certificar su discapacidad para acceder a beneficios estatales debe someterse a una evaluación médica y psicológica; en definitiva, el modelo permea todos los ámbitos de la discapacidad (Archer, 2009: 233-337). Ahora, la pregunta es ¿cómo una persona con discapacidad puede asumir su rol en la estructura del sistema médico, que parece opresivo y dominante, y al mismo tiempo sacarle ventajas?

Lo anterior se expresa en las personas con discapacidad de la siguiente forma; a una persona podría eventualmente "acomodarle" el sistema y la forma en que es intervenido. Por ejemplo; estar médicamente institucionalizado le permite acceder a un diagnóstico que lo certifica en la condición de discapacidad, y puede optar a beneficios diferenciados y a tratos preferentes (un estatus reconocido, en el lenguaje de Weber) por parte del Estado (pensiones, subsidios, ayudas técnicas, financiación de proyectos, estacionamientos exclusivos, etc.). El individuo se conforma con su rol en la estructura, no necesariamente renunciando a su posibilidad de ser sujeto, debido a que, a partir de los recursos e instancias ofertadas por el Estado como sistema estructural, reclama para sí la posibilidad ser más incluido, aumentando los espacios de participación y reconocimiento en la vida social y, sobre todo, adquiriendo más autonomía en las decisiones de su vida.

\section{Reflexiones finales}

Podemos concluir que las aproximaciones conceptuales de la teoría sociológica clásica son vigentes, pertinentes y útiles para explicar/comprender el fenómeno de la discapacidad, sus causas e implicaciones. Al mismo tiempo, aparentemente nos enfrentamos a la discapacidad como una condición fuertemente constreñida por las estructuras económicas-productivas, por el Estado y, sobre todo, por un modelo paradigmático como lo es el médico, el cual se erige como hegemónico en temas de discapacidad.

Por otro lado, al acercarnos a los teóricos que enfatizan en la comprensión interpretativa de la acción social como Weber, tampoco vislumbramos mucha esperanza en la posibilidad de autonomía de las personas con discapacidad, ya que se dan procesos de dominación racional y tradicional por parte de las personas, que terminan naturalizando su condición, conformándose con su rol y orientando el sentido de su acción solo a las posibilidades permitidas por las estructuras que los modelan.

Una de las cosas que podemos observar desde las teorías sociológicas clásicas refiere a la relación dialéctica entre las estructuras objetivas que hacen a un sujeto (o las posibilidades de serlo) y sus expectativas 
subjetivas. "La dialéctica de las expectativas subjetivas y de las oportunidades objetivas opera por doquier en el mundo social y en la mayoría de los casos tiende a asegurar el ajuste de las primeras a las segundas" (Ferrante y Ferreira, 2011: 92).

Si bien en Giddens y Archer pudimos ver cómo los sujetos se apropian de la estructura, la utilizan, y le dan un sentido que eventualmente puede ser morfogenético (que produce transformaciones), no es menos cierto que por el momento habría que asumir, desde lo teórico y lo empírico, que las personas con discapacidad son más bien "construidas" por las estructuras del Estado y el sistema médico, aunque no definitivamente, siempre se puede pensar, a propósito de las aperturas inclusivas en nuestra sociedad, que puedan ellos mismos ser los arquitectos de su destino.

En otras palabras, la sociedad como sistema abierto siempre es susceptible de que las personas sean, según palabras textuales de Archer "capaces de resistir, repudiar, suspender o dar un rodeo por las tendencias estructurales y culturales de impredecibles maneras en razón de sus poderes creadores como seres humanos" (2009: 272). La oposición activa a un sistema social y sus respectivas posiciones asignadas es un asunto socioculturalmente contingente y no de inevitabilidad sistémica.

Lo cierto es que la teoría sociológica clásica, y los trabajos actuales en discapacidad que la han incorporado, revelan la importancia y el potencial explicativo/analítico de esta para tensionar los enfoques sobre la discapacidad; a la vez que objetivar el fenómeno de la discapacidad a través de la lente de la teoría sociológica nos permite identificar a los sujetos y colectivos con esta condición como actores enmarcados dentro de estructuras sociales y, por lo tanto, como "ocupantes" de posiciones sociales al mismo tiempo que como agentes pletóricos de sentido, capaces de significar sus experiencias y de reflexionar sobre sus relaciones y posiciones en el mundo social, en tanto productores de sentidos existenciales irrenunciables. 


\section{Referencias bibliográficas}

Abberley, P. (1987). "The concept of opression and the development of a social theory of disability". Disability, Handicap \& Society, 2, pp. 5-19.

Almeida, M. E. et al. (2008). Políticas en discapacidad y producción de sujetos. El papel del Estado. Ponencia presentada en las V Jornadas Nacionales Universidad y Discapacidad, San Miguel de Tucumán, Argentina.

Archer, M. (2009). Teoría social realista: el enfoque morfogenético. Chile: Ediciones Universidad Alberto Hurtado.

Bourdieu, P. (1991). El sentido práctico. Madrid: Taurus.

Bourdieu, P. (1998). La distinción. Madrid: Taurus. (Trabajo original publicado en 1979)

Bourdieu, P. (1999). Meditaciones pascalianas. Barcelona: Anagrama.

Bourdieu, P. (2000). Poder, derecho y clases sociales. Bilbao: Desclée de Brouwer.

Brogna, P. (2006). El nuevo paradigma de la discapacidad y el rol de los profesionales de la rehabilitación. Argentina: El Cisne.

Córdoba, P. (2008). Discapacidad y exclusión social: propuesta teórica de vinculación paradigmática. Panamá: Centro de Estudios Latinoamericanos Justo Arosemena - CELA. Recuperado de http://bibliotecavirtual.clacso.org.ar/Panama/cela/20120717104229/discapacidad.pdf

Corker, M. (2008). "Diferencias, fusiones y bases ¿son estos los límites de la representación teórica "precisa" de las experiencias de las personas con discapacidad?". En L. Barton (comp.), Superar las barreras de la discapacidad (pp. 124-141). Madrid: Morata.

Durkheim, E. (1987). La división social del trabajo. Madrid: Akal. (Trabajo original publicado en 1893)

Durkheim, E. (2000). Las reglas del método sociológico. México: Quinto Sol.

Ferreira, M. A. (2009). “De la minus-valía a la diversidad funcional: un nuevo marco teórico-metodológico”. Política y Sociedad, 47(1), pp. 45-65.

Ferrante, C. y Ferreira, M. A. (2011). "Cuerpo y habitus: el marco estructural de la experiencia de la discapacidad". Intersticios. Revista Sociológica de Pensamiento Crítico, 5(2), pp. 85-101.

Ferrante, C. (2008). "Cuerpo, discapacidad y posición social: una aproximación indicativa al habitus de la discapacidad en Argentina”. Intersticios. Revista Sociológica de Pensamiento Crítico, 2(1), pp. 173-185.

Ferreira, M. A. V. y Rodríguez Caamaño, M.J. (2008). "Sociología de la discapacidad: una propuesta teórica critica". Nómadas. Revista critica de ciencias sociales y jurídicas, 13(1), pp. 243-249.

Foucault, M. (2007). Nacimiento de la bio-política (Traductor Pons, Horacio). Buenos Aires: FCE (1979)

García-Alonso, J. V. (coord.) (2003). El movimiento de vida independiente. Experiencias Internacionales. Madrid: Fundación Luis Vives.

Giddens, A. (2011). La constitución de la sociedad. Bases para la teoría de la estructuración. Buenos Aires: Amorrortu Editores. (Trabajo original publicado en 1984).

Gómez-Tagle, E. y Castillo, D. (2016). "Sociología de la discapacidad”. Tla-melaua, 10(40), pp. 77-194. 
Imrie, R. (2001). "Barriered and bounded places and the spatialities of disability". Urban Studies, 18, pp. $231-237$. Imrie, R. (2004). "Disability, embodiment and the meaning of the home". Housing Studies, 19(5), pp. 745-64.

Marx, K. (1997). Introducción general a la crítica de la economía política. México: Siglo XX. (Trabajo original publicado en 1857)

Míguez, M.N. (2014). "Discapacidad como construcción social en Francia y Uruguay”. Revista chilena de terapia ocupacional, 14(2), pp. 61-70.

Oliver, M. (1990). The Politics of Disablement. London: The MacMillan Press.

Organización Internacional del Trabajo (OIT) (2001). Discapacidad y empleo [artículo en web]. Recuperado de https://www.un.org/development/desa/disabilities-es/discapacidad-y-empleo.html

Organización de las Naciones Unidas (ONU) (2017). Algunos datos sobre las personas con discapacidad [artículo en web]. Recuperado de https://www.un.org/development/desa/disabilities-es/algunos-datos-sobre-las-personas-con-discapacidad.html

Organización de las Naciones Unidas para la Educación, la Ciencia y la Cultura (UNESCO) (2013). Situación educativa de América Latina y el Caribe: hacia la educación de calidad para todos al 2015. Santiago de Chile: Oficina Regional de Educación para América Latina y el Caribe. Recuperado de http://www.unesco.org/new/ fileadmin/MULTIMEDIA/FIELD/Santiago/images/SITIED-espanol.pdf

Organización Mundial de la Salud (OMS) (2001). Clasificación Internacional del Funcionamiento, de la Discapacidad y de la Salud: versión abreviada. Madrid: Instituto de Mayores y Servicios Sociales (IMSERSO). Recuperado de http://apps.who.int/iris/bitstream/10665/43360/1/9241545445_spa.pdf

Pérez, M. E. y Chhabra, G. (2019). "Modelos teóricos de discapacidad: un seguimiento del desarrollo histórico del concepto de discapacidad en las últimas cinco décadas". Revista Española de Discapacidad, 7(I), pp. 7-27.

Romañach, J. y Palacios, A. (2008). "El modelo de la diversidad. Una nueva visión de la bioética desde la perspectiva de las personas con diversidad funcional (discapacidad)". Intersticios. Revista Sociológica de Pensamiento Crítico, 2(2), pp. 37-47.

Rosato, A. et al. (2009). "El papel de la ideología de la normalidad en la producción de discapacidad". Ciencia, docencia y tecnología, 39(20), pp. 87-105.

Schütz, A y Luckmann, T. (2003). Las estructuras del mundo de la vida. Buenos Aires: Amorrortu.

Servicio Nacional de la Discapacidad, SENADIS (2015). I/ Estudio Nacional de la Discapacidad 2015. Santiago de Chile: Servicio Nacional de la Discapacidad. Recuperado de: http://observatorio.ministeriodesarrollosocial. gob.cl/endisc/docs/Libro_Resultados_II_Estudio_Nacional_de_la_Discapacidad.pdf

Simmel, G. (2005). "La metrópolis y la vida mental”. Bifurcaciones. Revista de Estudios Culturales y Urbanos, 4, pp. 1-10. (Trabajo original publicado en 1903). Recuperado de www.bifurcaciones.cl/004/bifurcaciones_004_ reserva.pdf.

Solsona, D. (diciembre, 2017). Los imaginarios sociales de la discapacidad: la circulación de imágenes, rótulos y heteronomías en la región de Magallanes Chile. Comunicación presentada en XXXI Congreso de la Asociación Latinoamericana de Sociología, Montevideo, Uruguay. Recuperado de http://alas2017.easyplanners.info/opc/ tl/0219_diego_alfredo_solsona.pdf.

Solsona, D. et al. (2018). "Imaginarios sociales sobre la discapacidad en la región de Magallanes, Chile: de sutiles semánticas a prácticas instituidas”. Intersticios. Revista Sociológica de Pensamiento Crítico, 12(2), pp. 95-108. 
Valenzuela, A. M. (2015). "Interpelaciones desde la diversidad funcional. La (in) justa medida de la capacidad en Chile". Revista Española de Discapacidad, 3(2), pp. 55-77.

Venturiello, M. P. (2010). "El 'habitus discapacidad' a la luz de un relato de vida". Intersticios. Revista Sociológica de Pensamiento Crítico, 4(2), pp. 175-185.

Weber, M. (2002). Economía y Sociedad. México: Fondo de Cultura Económica. (Trabajo original publicado en 1922) 